\title{
Markovian Dynamics of Josephson Parametric Amplification
}

\author{
Waldemar Kaiser, Michael Haider, Johannes A. Russer, Peter Russer, and Christian Jirauschek \\ Institute for Nanoelectronics, Technical University of Munich, Arcisstraße 21, 80333 Munich, Germany \\ Correspondence to: Waldemar Kaiser (waldemar.kaiser@tum.de) and Christian Jirauschek (jirauschek@tum.de)
}

Received: 23 December 2016 - Revised: 25 August 2017 - Accepted: 6 September 2017 - Published: 21 September 2017

\begin{abstract}
In this work, we derive the dynamics of the lossy DC pumped non-degenerate Josephson parametric amplifier (DCPJPA). The main element in a DCPJPA is the superconducting Josephson junction. The DC bias generates the AC Josephson current varying the nonlinear inductance of the junction. By this way the Josephson junction acts as the pump oscillator as well as the time varying reactance of the parametric amplifier. In quantum-limited amplification, losses and noise have an increased impact on the characteristics of an amplifier. We outline the classical model of the lossy DCPJPA and derive the available noise power spectral densities. A classical treatment is not capable of including properties like spontaneous emission which is mandatory in case of amplification at the quantum limit. Thus, we derive a quantum mechanical model of the lossy DCPJPA. Thermal losses are modeled by the quantum Langevin approach, by coupling the quantized system to a photon heat bath in thermodynamic equilibrium. The mode occupation in the bath follows the Bose-Einstein statistics. Based on the second quantization formalism, we derive the Heisenberg equations of motion of both resonator modes. We assume the dynamics of the system to follow the Markovian approximation, i.e. the system only depends on its actual state and is memory-free. We explicitly compute the time evolution of the contributions to the signal mode energy and give numeric examples based on different damping and coupling constants. Our analytic results show, that this model is capable of including thermal noise into the description of the DC pumped non-degenerate Josephson parametric amplifier.
\end{abstract}

\section{Introduction}

Recent progress in fabrication of nanoelectronic devices and low-temperature physics has increased the interest in superconducting quantum circuits. As Bardeen et al. (1957) out- lined in the Bardeen Cooper Schrieffer (BCS) theory, superconductivity is based on the condensation of Cooper pairs. London (1961) introduced a macroscopic theory by describing the superconducting phase by coherent matter waves, which exhibits macroscopic quantum effects. Thus, fluctuations are very small, making superconducting quantum circuits interesting for low noise devices. The basic element in superconducting quantum circuits is the Josephson junction predicted by Josephson (1962). The Josephson effect predicts the tunneling of Cooper pairs in two weakly coupled superconductors. Weak coupling is achieved by a thin separating tunnel barrier (Anderson and Rowell, 1963; Russer and Russer, 2011). Josephson junction based devices require a theoretical treatment using quantum mechanics, as soon as the energy of a considered signal becomes as low as a few microwave energy quanta, i.e. photons, at very low temperatures of only a few Kelvin. Compared to a pure classical treatment, a quantum formalism also considers effects due to spontaneous emission as well as induced quantum noise by coupling the system to a Langevin heat bath.

Parametric amplification plays an important role in several physical phenomena and is also utilized for amplification of electric signals. The parametric amplifier amplifies the oscillating signal mode by coupling the mode to an idler mode (Blackwell and Kotzebue, 1961; Mollow and Glauber, 1967; Roy and Devoret, 2016). Strong coupling is achieved by an oscillating non-linear coupling parameter. Radio-frequency and microwave signals containing few quanta are weak compared to the noise level of most detectors (Mollow and Glauber, 1967). Recent experiments by Macklin et al. (2015) show, that parametric amplification based on the Josephson junction faces quantum-limited amplification. So far, Josephson parametric amplifiers (JPA) have been treated classically by Russer (1969) and Russer (1971). A quantum mechanical model of the ideal JPA is derived by Russer and Russer (2011), which is based on the model introduced by Louisell 
(1964). Josephson parametric amplifiers operating close to the quantum limit also require a quantum mechanical treatment of losses.

In this work, we analyze the DC pumped non-degenerate Josephson parametric amplifier (DCPJPA). Based on a classical description of the lossy DCPJPA, the current noise correlation and the spectral power densities are derived. As the classical model is not capable of describing all observable features of the quantum-limited amplification regime, as e.g. spontaneous emission, there is the need of a quantum mechanical description of the DCPJPA. Thus, we derive a quantum mechanical model of the circuit including noise and losses. Losses are considered using the quantum Langevin method, as outlined by Gardiner and Zoller (2010) and Roy and Devoret (2016). The resonator circuits, i.e. the signal and the idler circuit, are coupled to a heat bath, represented by a photon gas in thermal equilibrium. The heat bath induces fluctuations in the resonator modes and causes damping of the signal energy. The time evolution of the signal energy and the noise contributions are derived based on the Heisenberg equations of motion. Simplification of the Josephson coupling Hamiltonian is obtained by the rotating wave approximation (RWA). Markovian dynamics neglecting memoryeffects are assumed, which induce white noise into the circuit (Farias et al., 2009). We explicitly compute the time evolution of the energy contributions for different initial setting. Our analytic results show, that the considered quantum mechanical model is capable of including thermal noise into the description of the Josephson parametric amplifier. For low damping, the signal energy is also amplified exponentially as shown in previous publications by Russer and Russer (2012), damping reduces the amplification of the energy contributions. Furthermore, we derive the quantum mechanical current noise correlation resulting from the coupling to the heat bath and link it to the classical conductance.

The quantum mechanical treatment of a lossy DCPJPA can be very useful when it comes to detecting e.g. single microwave photons, where one is interested in the quantum noise added by the amplification process. Standard solidstate based amplifiers cannot be used at such low energies, because of their relatively high thermal noise, compared to superconducting parametric amplifiers. This could enable interesting applications in superconducting quantum computing, where single radio-frequency or microwave photons interact with qubits, themselves consisting of Josephson elements. The theoretical treatment of noise brought in by the environment is crucial for the realization and operation of superconducting quantum circuit based systems.

However, there are still a lot of things left open in the following discussions. First of all, the Josephson junction is considered ideal in the following, i.e. it is modeled as an ideal tunnel junction, without any effective dissipation mechanisms (Kirtley et al., 1988). Furthermore, a Markovian assumption is made on the heat bath coupling mechanism in the signal and idler modes of the considered amplifier sys- tem, i.e. the coupling of the system and the heat bath only depends on the current state.

\section{The Josephson Effect}

Bardeen et al. (1957) provided the theory, that superconductivity originates from the pairing of electrons with opposite spin and wave vector to Cooper pairs. The superconducting ground state can be described by a macroscopic matter wave function given by Feynman et al. (1965). The Josephson effect is observed in two weakly coupled superconductors, seperated by a thin tunnel barrier (Josephson, 1962; Russer and Russer, 2011). The superconducting tunneling current $i_{\mathrm{J}}(t)$ is described by the first Josephson equation given by

$i_{\mathrm{J}}(t)=I_{\mathrm{c}} \sin \varphi(t)$,

where $I_{\mathrm{c}}$ is the critical Josephson current and $\varphi(t)$ is the quantum phase difference between both superconductors. The second Josephson equation relates the quantum phase difference $\varphi$ to the applied voltage over the Josephson junction $v(t)$ by

$\frac{\partial \varphi}{\partial t}=\frac{2 \pi}{\Phi_{0}} v(t)$

with the magnetic flux quantum

$\Phi_{0}=\frac{h}{2 e} \approx 2.0678 \times 10^{-15} \mathrm{Vs}$,

with the elementary charge $e$ and the Planck's constant $h$. A DC voltage $V_{0}$ applied to the junction gives rise to an $\mathrm{AC}$ current oscillating with the Josephson frequency

$f_{0}=\frac{2 e V_{0}}{h}=483.6 \cdot V_{0} \frac{\mathrm{GHz}}{\mathrm{mV}}$,

with the elementary charge $e$ and the Planck's constant $h$. Introducing the magnetic flux $\Phi(t)$ as the integral of the voltage $v(t)$ over time, the energy $w_{\mathrm{J}}(t)$ stored in a Josephson junction is given by

$w_{\mathrm{J}}(\Phi(t))=W_{\mathrm{J}}\left[1-\cos \frac{2 \pi \Phi(t)}{\Phi_{0}}\right]$,

with the maximum Josephson energy $W_{\mathrm{J}}=\Phi_{0} I_{\mathrm{J}} / 2 \pi$. Thus, the ideal Josephson junction is non-dissipative. The energy can be considered as stored in the non-linear, lossless timevariable Josephson inductance $L_{\mathrm{S}}(t)$ defined by

$L_{\mathrm{S}}(t)=\frac{\Phi_{0}}{2 \pi I_{\mathrm{c}} \cos (\varphi(t))}$.

Different from other inductors, it is possible to apply a DC voltage to the Josephson junction. Although the flux $\Phi(t)$ and with it the quantum phase difference $\varphi(t)$ are going to infinity with time when a DC voltage is applied, according 


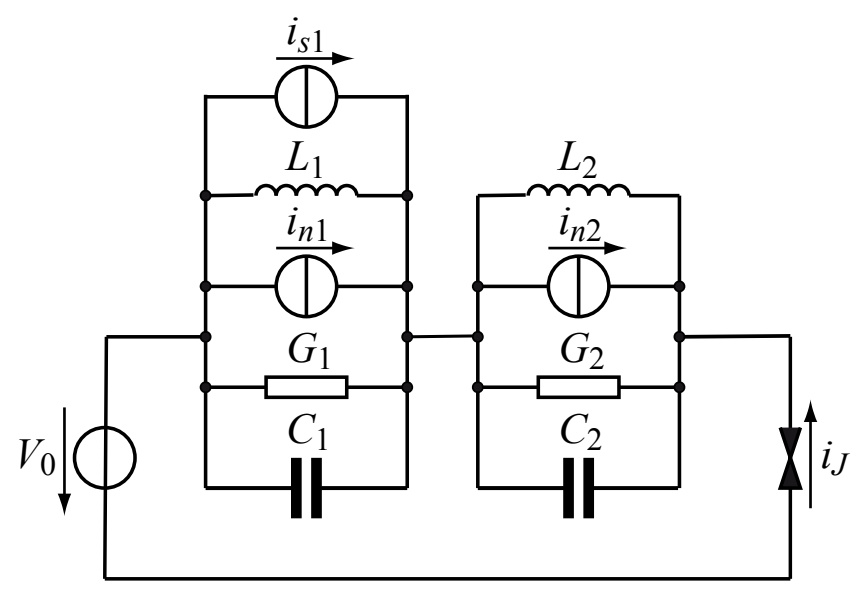

Figure 1. Equivalent circuit of the lossy DCPJPA.

to Eq. (5) the energy $w_{\mathrm{J}}(t)$ stored in a Josephson junction remains bounded. When a DC voltage is applied to the Josephson junction $L_{\mathrm{S}}^{-1}(t)$ varies sinusoidally with time. Hence, the Josephson junction can be used as the required nonlinear time-variable susceptance for parametric amplification as shown by Russer (1969) and Russer (1971).

\section{Classical Model of the Lossy Josephson Parametric Amplifier}

In this section, we outline the classical model of the lossy DCPJPA described by Russer (1969). The negative resistance three-frequency DCPJPA consists of a DC pumped Josephson junction oscillating with a frequency $f_{0}$ according to Eq. (4), coupled to two resonator modes with resonant frequencies $f_{10}$ and $f_{20}$ such that $f_{10}+f_{20}=f_{0}$ is fulfilled. Coupling a signal with frequency $f_{1} \approx f_{10}$ into the signal circuit by mixing in the Josephson junction in the idler circuit a signal at frequency $f_{2}$ is excited fulfilling $f_{1}+f_{2}=f_{0}$.

The equivalent circuit of the DCPJPA is shown in Fig. 1. The inductor $L_{1}$ and the capacitor $C_{1}$ constitute the signal resonator with resonant frequency $f_{10}, L_{2}$ and $C_{2}$ represent the idler resonator mode with resonant frequency $f_{20}$. Classically, losses are modeled by conductances $G_{1}$ and $G_{2}$. The thermal noise generated by the conductances $G_{1}$ and $G_{2}$ is represented by the equivalent noise current sources $i_{n 1}(t)$ and $i_{n 2}(t)$ and $i_{s 1}(t)$ is the signal current source.

The relation between signal, idler and pump frequency is crucial for the device characteristics. The general energy relations of the circuit shown in Fig. 1 are derived by Russer (1971). If the junction is biased by a DC voltage $V_{0}$ such that $f_{0}=f_{10}+f_{20}$, the signal, idler and pump energies obey the Manley-Rowe relation, derived by Manley and Rowe (1956), including an additional DC term according to Russer (1971)

$\frac{P_{1}}{f_{1}}=\frac{P_{2}}{f_{2}}=-\frac{P_{0}}{f_{1}+f_{2}}$.
This energy relation indicates the possibility of nondegenerate DC-pumped parametric amplification. Other choices of the relation between the different mode frequencies and the pump frequency can result in other operational modes such as a degenerate parametric amplifier or an upconverter (Russer and Russer, 2011). Since the power flow at $f_{1}$ and $f_{2}$ has opposite direction of the DC power flow at $f_{0}$, the Josephson junction impedance at $f_{1}$ and $f_{2}$ exhibits a negative real part, i.e. the magnitude of the reflection factor is greater than 1. In this case the DCPJPA acts as a negativeresistance reflection amplifier at $f_{1}$. When the signal is coupled out at $f_{2}$ an additional power gain $f_{2} / f_{1}$ is obtained.

For the lossy DCPJPA the classical equations of motion are given by

$$
\begin{aligned}
\frac{\mathrm{d} Q_{1}(t)}{\mathrm{d} t} & =\frac{\Phi_{1}(t)}{L_{1}}-G_{1} \frac{Q_{1}(t)}{C_{1}}+i_{s 1}(t)+i_{n 1}(t) \\
& +I_{\mathrm{c}} \sin \left(\omega_{0} t\right)+\frac{2 e I_{\mathrm{c}}}{\hbar}\left(\Phi_{1}(t)+\Phi_{2}(t)\right) \cos \left(\omega_{0} t\right), \\
\frac{\mathrm{d} Q_{2}(t)}{\mathrm{d} t} & =\frac{\Phi_{2}(t)}{L_{2}}-G_{2} \frac{Q_{2}(t)}{C_{2}}+i_{n 2}(t) \\
& +I_{\mathrm{c}} \sin \left(\omega_{0} t\right)+\frac{2 e I_{\mathrm{c}}}{\hbar}\left(\Phi_{1}(t)+\Phi_{2}(t)\right) \cos \left(\omega_{0} t\right), \\
\frac{\mathrm{d} \Phi_{1}(t)}{\mathrm{d} t} & =-\frac{Q_{1}(t)}{C_{1}} \\
\frac{\mathrm{d} \Phi_{2}(t)}{\mathrm{d} t} & =-\frac{Q_{2}(t)}{C_{2}} .
\end{aligned}
$$

The angular frequency $\omega_{0}=2 \pi f_{0}$ is determined via Eq. (4) by the applied DC voltage $V_{0}$.

The conductances $G_{1}$ and $G_{2}$ yield a decay of the signals in the signal- and idler circuits. The thermal excitation does not decay, since it is regenerated due to the dissipationfluctuation theorem (de Groot and Mazur, 1969, p. 151) by thermal fluctuations in the conductances. Considering the thermal noise sources $i_{n 1}(t)$ and $i_{n 2}(t)$ as classical Langevin noise sources, see (Haus, 2000, p. 143), they exhibit the correlation functions $c_{11}(\tau)$ and $c_{22}(\tau)$, respectively

$c_{11}(\tau) \equiv\left\langle i_{n 1}(t) i_{n 1}\left(t^{\prime}\right)\right\rangle=\frac{4 k_{B} T}{G_{1}} \delta\left(t-t^{\prime}\right)$,
$c_{22}(\tau) \equiv\left\langle i_{n 2}(t) i_{n 2}\left(t^{\prime}\right)\right\rangle=\frac{4 k_{B} T}{G_{2}} \delta\left(t-t^{\prime}\right)$,

where $\langle\ldots\rangle$ denotes the ensemble average, $k_{B}$ is the Boltzmann constant and $T$ is the absolute temperature of the lossy resonator.

The parametric amplification of the DCPJPA according to Fig. 1 is treated in detail in Russer (1969). There the maximum available power gains $g_{\mathrm{p} 11 \max }$ and $g_{\mathrm{p} 22 \max }$ of the negative resistance parametric amplifier operated at frequencies $f_{1}$ and $f_{2}$, respectively, the maximum available power gains $g_{\mathrm{p} 21 \max }$ of the up-converter and $g_{\mathrm{p} 12 \max }$ of the down- 
converter are obtained following Russer (1969) as

$$
\begin{aligned}
& g_{\mathrm{p} 11 \max }=g_{\mathrm{p} 22 \max }=\frac{4}{(1-\eta)^{2}}, \\
& g_{\mathrm{p} 12 \max }=\frac{f_{1}}{f_{2}} \frac{4 \eta}{(1-\eta)^{2}}, \\
& g_{\mathrm{p} 21 \max }=\frac{f_{2}}{f_{1}} \frac{4 \eta}{(1-\eta)^{2}},
\end{aligned}
$$

with

$\eta=\frac{e^{2} I_{\mathrm{c}}^{2}}{h^{2} f_{1} f_{2} G_{1} G_{2}}$.

To achieve high gain $\eta$ has to be close to 1 . If $\eta$ reaches 1 , the parametric amplifier becomes unstable. We assumed single conductances $G_{1}$ and $G_{2}$ in the signal and idler circuits, respectively. Splitting the $G_{1}$ into a generator conductance, a loss conductance and a load conductance reduces the gain, see Russer (1969) and Blackwell and Kotzebue (1961).

The Fourier transform of Eqs. (9a) and (9b) yields the autocorrelation spectra of the noise current sources

$C_{11}(\omega)=\frac{2 k_{B} T}{\pi G_{1}}$,
$C_{22}(\omega)=\frac{2 k_{B} T}{\pi G_{2}}$.

The available noise power spectral densities of $G_{1}$ and $G_{2}$, respectively are

$C^{P n a}=\frac{1}{2 \pi} k_{B} T$.

with Eqs. (10a) to (10c) this yields the spectral power densities $C^{\mathrm{Pn} 1}$ and $C^{\mathrm{Pn} 2}$ in the signal and idler circuits,

$C^{\mathrm{Pn} 1}(\omega)=\frac{2}{\pi}\left[\frac{1}{(1-\eta)^{2}}+\frac{f_{1}}{f_{2}} \frac{1 \eta}{(1-\eta)^{2}}\right] k_{B} T$,

$C^{\mathrm{Pn} 2}(\omega)=\frac{2}{\pi}\left[\frac{1}{(1-\eta)^{2}}+\frac{f_{2}}{f_{1}} \frac{1 \eta}{(1-\eta)^{2}}\right] k_{B} T$.

In the classical limit the idler noise contribution to the noise in the signal circuit is down-converted by a factor $f_{1} / f_{2}$.

We obtain the semiclassical approach for the quantum noise when replacing

$k_{B} T \rightarrow \frac{h f}{1-\exp \frac{h f}{k T}}$.
Inserting this into Eqs. (14a) and (14b) yields

$$
\begin{aligned}
& C^{\operatorname{Pn} 1}(\omega)=\frac{2 h f_{1}}{\pi(1-\eta)^{2}}\left[\frac{1}{1-\exp \frac{h f_{1}}{k T}}+\frac{\eta}{1-\exp \frac{h f_{2}}{k T}}\right], \\
& C^{\operatorname{Pn} 2}(\omega)=\frac{2 h f_{2}}{\pi(1-\eta)^{2}}\left[\frac{1}{1-\exp \frac{h f_{2}}{k T}}+\frac{\eta}{1-\exp \frac{h f_{1}}{k T}}\right] .
\end{aligned}
$$

In the limit $h f \ll k T$ these equations yield Eqs. (14a) and (14b) whereas in the limit $h f \gg k T$ we obtain

$$
\begin{aligned}
& C^{\mathrm{Pn} 1}(\omega)=2 \frac{1+\eta}{\pi(1-\eta)^{2}} h f_{1}, \\
& C^{\mathrm{Pn} 2}(\omega)=2 \frac{1+\eta}{\pi(1-\eta)^{2}} h f_{2} .
\end{aligned}
$$

\section{Quantum Mechanical Model of the Lossy Josephson Parametric Amplifier}

In this section, we outline the quantum mechanical model of the lossy DCPJPA described by Russer and Russer (2011) and Jirauschek and Russer (2012).

Here, we have used a second order Taylor expansion for the energy stored in the nonlinear Josephson inductance. We expressed the equations of motion in terms of signal and idler charges and fluxes, since they form a set of conjugate variables which will be useful later, when stating the quantum mechanical Hamiltonian.

In a quantum mechanical model dissipation and fluctuations which are modeled in the classical model by the loss conductances $G_{1}$ and $G_{2}$ and the related noise current sources $i_{n 1}$ and $i_{n 2}$ are modeled by coupling the conservative quantum system formed by the capacitors, inductors and the Josephson junction to a so-called heat bath. This heat bath is a many-electron system of electrons in thermal equilibrium. The losses originate from the transfer of energy to this manyelectron system where the transferred energy is randomized. Vice-versa fluctuations from the heat bath are coupled into the resonant circuits as outlined by Walls and Milburn (2007) and Gardiner and Zoller (2010).

The total Hamiltonian of the lossy DCPJPA is given by

$\mathbf{H}=\mathbf{H}_{\mathrm{LC}}+\mathbf{H}_{J J}+\mathbf{H}_{\mathrm{B}}+\mathbf{H}_{\mathrm{C}}$

where the unperturbed Hamiltonian $\mathbf{H}_{\mathrm{LC}}$ represents the ideal lossless LC resonant circuits, the operator $\mathbf{H}_{J J}$ describes the energy of the DC biased Josephson junction, $\mathbf{H}_{\mathrm{B}}$ represents the heat bath describing a photon field in thermodynamic equilibrium and $\mathbf{H}_{\mathrm{C}}$ gives the coupling between the $\mathrm{LC}$ resonators and the heat bath. We apply the second quantization formalism outlined by Dirac (1927) and Fock (1932). We introduced the annihilation operator $\mathbf{a}_{i}$ and the creation opera- 
tor $\mathbf{a}_{i}^{\dagger}$ given by

$\mathbf{a}_{i}=\sqrt{\frac{1}{2 \hbar \omega_{i} L_{i}}} \boldsymbol{\Phi}_{i}+\mathrm{i} \sqrt{\frac{\omega_{i} L_{i}}{2 \hbar}} \mathbf{Q}_{i}$,

$\mathbf{a}_{i}^{\dagger}=\sqrt{\frac{1}{2 \hbar \omega_{i} L_{i}}} \boldsymbol{\Phi}_{i}-\mathrm{i} \sqrt{\frac{\omega_{i} L_{i}}{2 \hbar}} \mathbf{Q}_{i}$,

where $\hbar=h /(2 \pi)$ is the reduced Planck constant, and $\boldsymbol{\Phi}_{i}$ and $\mathbf{Q}_{i}$ are the flux and charge operators, respectively, of the $i$ th LC circuit. The flux and charge operators fulfill the canonical commutation relation

$\left[\boldsymbol{\Phi}_{i}, \mathbf{Q}_{j}\right]=\mathrm{i} \hbar \delta_{i j}$

which gives the bosonic commutation relation

$\left[\mathbf{a}_{i}, \mathbf{a}_{j}^{\dagger}\right]=\delta_{i j}$

With Eqs. (19a) and (19b) the system Hamiltionans are given by

$\mathbf{H}_{\mathrm{LC}}=\hbar \omega_{1}\left(\mathbf{a}_{1}^{\dagger} \mathbf{a}_{1}+\frac{1}{2}\right)+\hbar \omega_{2}\left(\mathbf{a}_{2}^{\dagger} \mathbf{a}_{2}+\frac{1}{2}\right)$,

$\mathbf{H}_{J J}=W_{\mathrm{J}}\left\{1-\cos \left[\omega_{0} t+\lambda_{1}\left(\mathbf{a}_{1}+\mathbf{a}_{1}^{\dagger}\right)+\lambda_{2}\left(\mathbf{a}_{2}+\mathbf{a}_{2}^{\dagger}\right)\right]\right\}$,

where the dimensionless parameter $\lambda_{i}$ of the $i$ th LC circuit coupled by the Josephson junction is defined by

$\lambda_{i}=\sqrt{\frac{2 \alpha}{\pi Z_{F 0} Z_{i}}}$.

Here, $\alpha$ is the Sommerfeld's constant given by

$\alpha=\frac{e^{2}}{2 c \varepsilon_{0} h} \approx \frac{1}{137.036}$.

$\epsilon_{0}$ is the vacuum permittivity, $c$ is the speed of light, $Z_{i}=$ $\sqrt{C_{i} / L_{i}}$ is the characteristic impedance of the $i$-th LC circuit, and $Z_{F 0}$ is the free-space wave impedance.

The rotating wave approximation (RWA) is commonly used in analytical treatments of quantum optical systems. According to Walls and Milburn (2007), the system perspective is changed into a rotating frame. Rapidly rotating terms are neglected, which delivers a valid approximation in case of near resonance terms and low intensities ( $\mathrm{Wu}$ and Yang, 2007). Applying the RWA for the DCPJPA yields

$\mathbf{H}_{J J}^{N R} \approx \gamma_{j}\left[\mathbf{a}_{1}^{\dagger} \mathbf{a}_{2}^{\dagger} \exp \left(-\mathrm{i} \omega_{0} t\right)+\mathbf{a}_{1} \mathbf{a}_{2} \exp \left(\mathrm{i} \omega_{0} t\right)\right]$,

where $\gamma_{j}=W_{\mathrm{J}} \lambda_{1} \lambda_{2}$ is the Josephson coupling parameter describing the coupling strength between both resonators.

The energy of the ideal DCPJPA rises exponentially with time, as derived by Walls and Milburn (2007), and Russer and Russer (2011), describing a parametric oscillation rather than a parametric amplification. Applying the Langevin method we can treat losses of quantum mechanical systems by coupling the system to a heat bath as proposed by Sargent et al. (1974) and Gardiner and Collett (1985). A heat bath is described by a large system of closely spaced harmonic oscillators in thermodynamic equilibrium. In Fig. 1 the heat bath modes are represented by dashed modes inside the frame. The Hamilton operators of the heat bath and the coupling between the system and the bath are given by

$$
\begin{aligned}
\mathbf{H}_{\mathrm{B}} & =\sum_{k} \hbar \widetilde{\omega}_{k}\left(\mathbf{b}_{k}^{\dagger} \mathbf{b}_{k}+\frac{1}{2}\right), \\
\mathbf{H}_{\mathrm{C}} & =\sum_{i=1}^{2} \sum_{k} \frac{1}{2} \hbar \kappa_{i k}\left(\mathbf{a}_{i} \mathbf{b}_{k}^{\dagger}+\mathbf{b}_{k} \mathbf{a}_{i}^{\dagger}\right),
\end{aligned}
$$

where $\mathbf{b}_{k}$ is the bath operator with angular resonance frequency $\widetilde{\omega}_{k}$. The coefficients $\kappa_{i k}$ are of microscopic origin, describing the coupling strength between the $i$ th resonator and the heat bath mode $k$. The Hamilton operator $\mathbf{H}_{\mathrm{C}}$ describes the so-called heat-bath, a large phonon system in thermal equilibrium. Energy from our conservative system under consideration (SUC) is coupled into the heat bath and dissipated by distribution over the phonon system. By this way the energy is randomized. Vice-versa, since the coupling between the SUC and the heat bath is reciprocal and obeys detailed balance, fluctuations are coupled from the heat bath into the SUC. This is stated by the fluctuationdissipation theorem which applies both to classical and quantum mechanical systems. Whereas in the classical consideration in the previous chapter dissipation and fluctuations simply could be modeled phenomenologically applying a conductance and an equivalent noise source, the quantum mechanical treatment requires a micro-physical model using the quantum mechanical many-body system of the heat bath. In Sect. 5, the relation between the coupling coefficients $\kappa_{i k}$ and the classical circuit parameters is derived.

\section{Quantum Langevin Equations}

The time evolution of the system operators is computed in the Heisenberg representation using the Heisenberg equations of motion

$$
\frac{\mathrm{d}}{\mathrm{d} t} \mathbf{O}_{\mathrm{H}}=\frac{\mathrm{i}}{\hbar}\left[\mathbf{H}_{\mathrm{H}}, \mathbf{O}_{\mathrm{H}}\right]
$$

where $\mathbf{O}_{\mathrm{H}}$ is any arbitrary operator describing the system. Using the Hamiltonian defined in Eq. (18), the Heisenberg 
equations of motion are given by

$$
\begin{aligned}
\frac{\mathrm{d} \mathbf{a}_{1 \mathrm{H}}}{\mathrm{d} t} & =-\mathrm{i} \omega_{1} \mathbf{a}_{1 \mathrm{H}}-\mathrm{i} \frac{\gamma_{j}}{\hbar} \mathbf{a}_{2 \mathrm{H}}^{\dagger} e^{-\mathrm{i} \omega_{0} t}-\mathrm{i} \sum_{k} \frac{\kappa_{1 k}}{2} \mathbf{b}_{k \mathrm{H}}, \\
\frac{\mathrm{d} \mathbf{a}_{2 \mathrm{H}}}{\mathrm{d} t} & =-\mathrm{i} \omega_{2} \mathbf{a}_{2 \mathrm{H}}-\mathrm{i} \frac{\gamma_{j}}{\hbar} \mathbf{a}_{1 \mathrm{H}}^{\dagger} e^{-\mathrm{i} \omega_{0} t}-\mathrm{i} \sum_{k} \frac{\kappa_{2 k}}{2} \mathbf{b}_{k \mathrm{H}}, \\
\frac{\mathrm{d} \mathbf{b}_{k \mathrm{H}}}{\mathrm{d} t} & =-\mathrm{i} \widetilde{\omega}_{k} \mathbf{b}_{k \mathrm{H}}-\mathrm{i} \sum_{i=1}^{2} \frac{\kappa_{i k}}{2} \mathbf{a}_{i \mathrm{H}} .
\end{aligned}
$$

Here, the bosonic commutator relations described by Louisell (1964) and Sargent et al. (1974) are applied. As we are only interested in the dynamics of the circuit operators, we eliminate the bath degrees of freedom by integrating Eq. (29c). Hereby, we obtain

$$
\begin{aligned}
\mathbf{b}_{k \mathrm{H}}(t) & =\mathbf{b}_{k \mathrm{H}}(0) \mathrm{e}^{-\mathrm{i} \widetilde{\omega}_{k}\left(t-t_{0}\right)} \\
& -\mathrm{i} \sum_{i=1}^{2} \frac{\kappa_{i k}}{2} \int_{t_{0}}^{t} \mathbf{a}_{i \mathrm{H}}\left(t^{\prime}\right) \mathrm{e}^{-\mathrm{i} \widetilde{\omega}_{k}\left(t-t^{\prime}\right)} \mathrm{d} t^{\prime} .
\end{aligned}
$$

Replacing the integration variable $t-t^{\prime}=\tau$ yields

$$
\begin{aligned}
\mathbf{b}_{k \mathrm{H}}(t) & =\mathbf{b}_{k \mathrm{H}}(0) \mathrm{e}^{-\mathrm{i} \widetilde{\omega}_{k}\left(t-t_{0}\right)} \\
& -\mathrm{i} \sum_{i=1}^{2} \frac{\kappa_{i k}}{2} \int_{0}^{t-t_{0}} \mathbf{a}_{i \mathrm{H}}(t-\tau) \mathrm{e}^{-\mathrm{i} \widetilde{\omega}_{k} \tau} \mathrm{d} \tau .
\end{aligned}
$$

The initial time $t_{0}$ is set to zero without loss of generality. Substituting Eq. (31) into Eq. (29a) gives

$$
\begin{aligned}
\frac{\mathrm{d} \mathbf{a}_{1 \mathrm{H}}(t)}{\mathrm{d} t} & =-\mathrm{i} \omega_{1} \mathbf{a}_{1 \mathrm{H}}(t)-\mathrm{i} \frac{\gamma_{j}}{\hbar} \mathbf{a}_{2 \mathrm{H}}^{\dagger}(t) \mathrm{e}^{-\mathrm{i} \omega_{0} t}+\mathbf{f}_{1}(t) \\
& -\sum_{k} \sum_{i=1}^{2} \frac{\kappa_{1 k}}{2} \frac{\kappa_{i k}}{2} \int_{0}^{t} \mathbf{a}_{i \mathrm{H}}(t-\tau) \mathrm{e}^{-\mathrm{i} \tilde{\omega}_{k} \tau} \mathrm{d} \tau .
\end{aligned}
$$

In this equation we have introduced the Langevin noise operator in terms of the initial bath operators

$\mathbf{f}_{i}(t)=-\mathrm{i} \sum_{k} \frac{\kappa_{i k}}{2} \mathbf{b}_{k \mathrm{H}}(0) \mathrm{e}^{-\mathrm{i} \widetilde{\omega}_{k} t}$

The operator $\mathbf{f}_{i}(t)$ represents a stochastic term with white noise and Gaussian properties as described in detail by Farias et al. (2009). In the following, we denote $\mathbf{b}_{k \mathrm{H}}(0)=\mathbf{b}_{k \mathrm{H}}$. We further simplify the equations of motion by changing to the slowly varying operators defined by Sargent et al. (1974)

$\mathbf{A}_{i \mathrm{H}}(t)=\mathrm{e}^{\mathrm{i} \omega_{i} t} \mathbf{a}_{i \mathrm{H}}(t)$
Equation (32) is given in the slowly varying frame by

$$
\begin{aligned}
\frac{\mathrm{d} \mathbf{A}_{1 \mathrm{H}}(t)}{\mathrm{d} t} & =-\mathrm{i} \frac{\gamma_{j}}{\hbar} \mathbf{A}_{2 \mathrm{H}}^{\dagger}(t)+\mathbf{f}_{1}(t) \mathrm{e}^{\mathrm{i} \omega_{1} t}-\sum_{k} \sum_{i=1}^{2} \frac{\kappa_{1 k}}{2} \frac{\kappa_{i k}}{2} \\
& \times \int_{0}^{t} \mathbf{A}_{i \mathrm{H}}(t-\tau) \mathrm{e}^{-\mathrm{i}\left(\widetilde{\omega}_{k}-\omega_{i}\right) \tau} \mathrm{e}^{\mathrm{i}\left(\omega_{1}-\omega_{i}\right) t} \mathrm{~d} \tau .
\end{aligned}
$$

This equation can be simplified by applying the Markovian approximation. As outlined by Farias et al. (2009), in the Markovian approximation the system depends only on its current state, thus we obtain

$$
\begin{aligned}
\frac{\mathrm{d} \mathbf{A}_{1 \mathrm{H}}(t)}{\mathrm{d} t} & \approx-\mathrm{i} \frac{\gamma_{j}}{\hbar} \mathbf{A}_{2 \mathrm{H}}^{\dagger}(t)+\mathbf{f}_{1}(t) \mathrm{e}^{\mathrm{i} \omega_{1} t}-\sum_{k} \sum_{i=1}^{2} \frac{\kappa_{1 k}}{2} \frac{\kappa_{i k}}{2} \\
& \times \mathbf{A}_{i \mathrm{H}}(t) \int_{0}^{t} \mathrm{e}^{-\mathrm{i}\left(\widetilde{\omega}_{k}-\omega_{i}\right) \tau} \mathrm{e}^{\mathrm{i}\left(\omega_{1}-\omega_{i}\right) t} \mathrm{~d} \tau .
\end{aligned}
$$

We use according to Sargent et al. (1974)

$\int_{0}^{t} \mathrm{~d} \tau \exp [-\mathrm{i}(\Omega-\omega) \tau]=\pi \delta(\Omega-\omega)-\mathcal{P}\left(\frac{\mathrm{i}}{\Omega-\omega}\right)$,

with the Cauchy principal part $\mathcal{P}$. Assuming a dense distribution of heat bath modes in the frequency spectrum, we can replace the summation over the modes by an integral over the density of states

$\sum_{k} \rightarrow \int_{0}^{\infty} \mathrm{d} \omega \mathcal{D}(\omega)$

In case of the DCPJPA, we assume a photon heat bath in thermal equilibrium described by the density of states

$\mathcal{D}(\omega)=\frac{V \omega^{2}}{\pi^{2} c^{3}}$,

with the volume of the heat bath $V$. Applying this relation to Eq. (36) we obtain the rate equations

$$
\begin{aligned}
\frac{\mathrm{d} \mathbf{A}_{1 \mathrm{H}}(t)}{\mathrm{d} t}= & -\frac{1}{2} \widetilde{\gamma}_{11} \mathbf{A}_{1 \mathrm{H}}(t)-\mathrm{i} \frac{\gamma_{j}}{\hbar} \mathbf{A}_{2 \mathrm{H}}^{\dagger}(t)+\mathbf{g}_{1}(t) \\
& -\frac{1}{2} \widetilde{\gamma}_{21} \mathbf{A}_{2 \mathrm{H}}(t) \mathrm{e}^{\mathrm{i}\left(\omega_{1}-\omega_{2}\right) t}, \\
\frac{\mathrm{d} \mathbf{A}_{2 \mathrm{H}}(t)}{\mathrm{d} t}= & -\frac{1}{2} \widetilde{\gamma}_{22} \mathbf{A}_{2 \mathrm{H}}(t)-\mathrm{i} \frac{\gamma_{j}}{\hbar} \mathbf{A}_{1 \mathrm{H}}^{\dagger}(t)+\mathbf{g}_{2}(t) \\
& -\frac{1}{2} \widetilde{\gamma}_{12} \mathbf{A}_{1 \mathrm{H}}(t) \mathrm{e}^{\mathrm{i}\left(\omega_{2}-\omega_{1}\right) t},
\end{aligned}
$$

with the noise operator $\mathbf{g}_{i}$ in the slowly varying frame defined by

$\mathbf{g}_{i}=\mathbf{f}_{i} \mathrm{e}^{\mathrm{i} \omega t}$. 
Hereby, we introduced the damping matrix $\widetilde{\gamma}_{i j}$ given by

$\tilde{\gamma}=\frac{\pi}{2}\left[\begin{array}{cc}\kappa_{1}^{2}\left(\omega_{1}\right) \mathcal{D}\left(\omega_{1}\right) & \kappa_{1}\left(\omega_{1}\right) \kappa_{2}\left(\omega_{1}\right) \mathcal{D}\left(\omega_{1}\right) \\ \kappa_{1}\left(\omega_{2}\right) \kappa_{2}\left(\omega_{2}\right) \mathcal{D}\left(\omega_{2}\right) & \kappa_{2}^{2}\left(\omega_{2}\right) \mathcal{D}\left(\omega_{2}\right)\end{array}\right]$.

If the noise operators $\mathbf{g}_{i}$ are uncorrelated, we can drop the off-diagonal damping terms $\widetilde{\gamma}_{i j}$. Experimentally, the diagonal damping terms $\tilde{\gamma}_{i i}$ are observable by measuring the linewidth of the resonance curve of the $i$ th LC resonator. Following Russer and Kaertner (1990), an input signal $\mathbf{b}_{\mathrm{S}}^{\text {in }}(t)$ can be introduced in the rate equations with the coupling factor

$\gamma_{\mathrm{S}}=\frac{1}{R_{\mathrm{S}} C}$,

with $R_{\mathrm{S}}$ as the impedance of the transmission line, from which the signal is coupled to the LC resonant mode.

We obtain the rate equations of the photon annihilation and creation operators

$$
\begin{aligned}
\frac{\mathrm{d} \mathbf{A}_{1 \mathrm{H}}(t)}{\mathrm{d} t}= & -\frac{1}{2} \widetilde{\gamma}_{11} \mathbf{A}_{1 \mathrm{H}}(t)-\mathrm{i} \frac{\gamma_{j}}{\hbar} \mathbf{A}_{2 \mathrm{H}}^{\dagger}(t)+\mathbf{g}_{1}(t) \\
& +\sqrt{\gamma_{\mathrm{S}}} \mathbf{b}_{\mathrm{S}}^{\mathrm{in}}(t), \\
\frac{\mathrm{d} \mathbf{A}_{1 \mathrm{H}}^{\dagger}(t)}{\mathrm{d} t}= & -\frac{1}{2} \widetilde{\gamma}_{11}^{*} \mathbf{A}_{1 \mathrm{H}}^{\dagger}(t)+\mathrm{i} \frac{\gamma_{j}^{*}}{\hbar} \mathbf{A}_{2 \mathrm{H}}(t)+\mathbf{g}_{1}^{\dagger}(t) \\
& +\sqrt{\gamma_{\mathrm{S}}} \mathbf{b}_{\mathrm{S}}^{\mathrm{in} \dagger}(t), \\
\frac{\mathrm{d} \mathbf{A}_{2 \mathrm{H}}(t)}{\mathrm{d} t}= & -\frac{1}{2} \widetilde{\gamma}_{22} \mathbf{A}_{2 \mathrm{H}}(t)-\mathrm{i} \frac{\gamma_{j}}{\hbar} \mathbf{A}_{1 \mathrm{H}}^{\dagger}(t)+\mathbf{g}_{2}(t), \\
\frac{\mathrm{d} \mathbf{A}_{2 \mathrm{H}}^{\dagger}(t)}{\mathrm{d} t}= & -\frac{1}{2} \widetilde{\gamma}_{22}^{*} \mathbf{A}_{2 \mathrm{H}}^{\dagger}(t)+\mathrm{i} \frac{\gamma_{j}^{*}}{\hbar} \mathbf{A}_{1 \mathrm{H}}(t)+\mathbf{g}_{2}^{\dagger}(t) .
\end{aligned}
$$

Following Russer and Kaertner (1990), the output field operator can be obtained by

$\mathbf{b}_{\mathrm{S}}^{\text {out }}(t)=\sqrt{\gamma_{\mathrm{S}}} \mathbf{A}_{1 \mathrm{H}}(t)-\mathbf{b}_{\mathrm{S}}^{\text {in }}(t)$.

In this work, we consider the input signal as an initial occupation of the signal mode and derive the dynamics of the internal amplifier mode $\mathbf{A}_{1 \mathrm{H}}$. According to Eq. (45), the output signal operator $\mathbf{b}_{\mathrm{S}}^{\text {out }}$ only depends on the signal mode operator $\mathbf{A}_{1 \mathrm{H}}$ for a known input $\mathbf{b}_{\mathrm{S}}^{\mathrm{in}}$. Thus, an increase of the expectation value in the signal operator $\mathbf{A}_{1 \mathrm{H}}$ or equivalently in the signal mode energy is required in order to amplify the input signal.
Solving Eqs. (44a)-(44d), not considering the input signal operator $\mathbf{b}_{\mathrm{S}}^{\text {in }}$ yields

$$
\begin{aligned}
& \mathbf{A}_{1 \mathrm{H}}(t)=\xi_{11}(t) \mathbf{A}_{1 \mathrm{H}}(0)+\xi_{12}(t) \mathbf{A}_{2 \mathrm{H}}^{\dagger}(0) \\
& +\sum_{k} \kappa_{1 k} \mathbf{b}_{k \mathrm{H}}\left[-\alpha_{+}\left(\widetilde{\omega}_{k}\right)+\alpha_{-}\left(\widetilde{\omega}_{k}\right)\right] \mathrm{e}^{\mathrm{i}\left(\omega_{1}-\widetilde{\omega}_{k}\right) t} \\
& +\sum_{k} \kappa_{1 k} \mathbf{b}_{k \mathrm{H}}\left[\alpha_{+}\left(\widetilde{\omega}_{k}\right) \mathrm{e}^{-\frac{\gamma t}{4}}-\alpha_{-}\left(\widetilde{\omega}_{k}\right) \mathrm{e}^{\frac{\gamma t}{4}}\right] \mathrm{e}^{-\frac{t}{4}\left(\widetilde{\gamma}_{11}+\widetilde{\gamma}_{22}^{*}\right)} \\
& +\sum_{k} \kappa_{2 k}^{*} \mathbf{b}_{k \mathrm{H}}^{\dagger}\left[-\beta_{+}\left(\widetilde{\omega}_{k}\right)+\beta_{-}\left(\widetilde{\omega}_{k}\right)\right] e^{-\mathrm{i}\left(\omega_{2}-\widetilde{\omega}_{k}\right) t} \\
& +\sum_{k} \kappa_{2 k}^{*} \mathbf{b}_{k \mathrm{H}}^{\dagger}\left[\beta_{+}\left(\widetilde{\omega}_{k}\right) e^{-\frac{\gamma t}{4}}-\beta_{-}\left(\widetilde{\omega}_{k}\right) e^{\frac{\gamma t}{4}}\right] e^{-\frac{t}{4}\left(\widetilde{\gamma}_{11}+\widetilde{\gamma}_{22}^{*}\right)},
\end{aligned}
$$

with

$$
\begin{aligned}
\xi_{11}(t) & =\left[\cosh \left(\frac{t \gamma}{4}\right)+\frac{\left(\tilde{\gamma}_{22}^{*}-\widetilde{\gamma}_{11}\right)}{\eta} \sinh \left(\frac{t \gamma}{4}\right)\right] \\
& \times e^{-\frac{t}{4}\left(\widetilde{\gamma}_{11}+\tilde{\gamma}_{22}^{*}\right)}, \\
\xi_{12}(t) & =-\frac{4 \gamma_{j} \mathrm{i}}{\gamma \hbar} \sinh \left(\frac{t \gamma}{4}\right) e^{-\frac{t}{4}\left(\widetilde{\gamma}_{11}+\widetilde{\gamma}_{22}^{*}\right)}, \\
\alpha_{ \pm}(\omega) & =\frac{\mathrm{i}}{\gamma} \frac{ \pm \gamma-\widetilde{\gamma}_{22}^{*}+\widetilde{\gamma}_{11}}{\widetilde{\gamma}_{11}+\widetilde{\gamma}_{22}^{*} \pm \gamma+4 \mathrm{i}\left(\omega_{1}-\omega\right)}, \\
\beta_{ \pm}(\omega) & =4 \frac{\gamma_{j}}{\hbar \gamma} \frac{1}{\widetilde{\gamma}_{11}+\widetilde{\gamma}_{22}^{*} \pm \gamma-4 \mathrm{i}\left(\omega_{2}-\omega\right)} .
\end{aligned}
$$

Here, we have introduced the effective driving parameter

$\gamma=\sqrt{\left(\widetilde{\gamma}_{11}-\widetilde{\gamma}_{22}^{*}\right)^{2}+16 \frac{\left|\gamma_{j}\right|^{2}}{\hbar^{2}}}$.

We can directly link the energy expectation value in the signal circuit to the expectation value of the number operator $\mathbf{n}_{L C, 1}$ by

$$
\left\langle E_{L C, 1}\right\rangle=\hbar \omega_{1}\left(\left\langle\mathbf{n}_{L C, 1}\right\rangle+1\right) .
$$

In analogy to Sargent et al. (1974) the computed expectation value of the number operator is described by

$$
\begin{aligned}
\left\langle\mathbf{n}_{\mathrm{LC}, 1}(t)\right\rangle & =\left\langle\mathbf{A}_{1 \mathrm{H}}^{\dagger}(t) \mathbf{A}_{1 \mathrm{H}}(t)\right\rangle \\
& =\left\langle\mathbf{n}_{\text {Signal }}(t)\right\rangle+\left\langle\mathbf{n}_{\text {Idler }}(t)\right\rangle+\left\langle\mathbf{n}_{\text {Noise }}(t)\right\rangle .
\end{aligned}
$$

The signal photon number is given by

$$
\left\langle\mathbf{n}_{\text {Signal }}(t)\right\rangle=\xi_{11}^{*}(t) \xi_{11}(t)\left\langle\mathbf{n}_{\text {Signal }}(0)\right\rangle .
$$

The photon number of the down-converted idler noise is described by

$\left\langle\mathbf{n}_{\text {Idler }}(t)\right\rangle=\xi_{12}^{*}(t) \xi_{12}(t)\left(\left\langle\mathbf{n}_{\text {Idler }}(0)\right\rangle+1\right)$, 
and the noise photon number rising from the coupling of the signal mode to the heat bath

$$
\begin{aligned}
& \left\langle\mathbf{n}_{\text {Noise }}(t)\right\rangle=2 \widetilde{\gamma}_{11} \bar{n}\left(\omega_{1}\right)\left|\alpha\left(\omega_{1}\right)\right|^{2}+2 \widetilde{\gamma}_{22}\left[\bar{n}\left(\omega_{2}\right)+1\right]\left|\beta\left(\omega_{2}\right)\right|^{2} \\
& +\widetilde{\gamma}_{22}\left[\bar{n}\left(\omega_{2}\right)+1\right] \frac{4}{\pi}\left[\beta\left(\omega_{2}\right)\left(e^{-\frac{\gamma t}{4}} \beta_{+}\left(\omega_{2}\right)-e^{\frac{\gamma t}{4}} \beta_{-}\left(\omega_{2}\right)\right)\right. \\
& \left.+\left|e^{-\frac{\gamma t}{4}} \beta_{+}\left(\omega_{2}\right)-e^{\frac{\gamma t}{4}} \beta_{-}\left(\omega_{2}\right)\right|^{2}\right] e^{-\frac{t}{4}\left(\widetilde{\gamma}_{11}+\widetilde{\gamma}_{22}^{*}\right)} \\
& +\widetilde{\gamma}_{11} \bar{n}\left(\omega_{1}\right) \frac{4}{\pi}\left\{\mathcal{R}\left[\alpha^{*}\left(\omega_{1}\right)\left(e^{-\frac{\gamma t}{4}} \alpha_{+}\left(\omega_{1}\right)-e^{\frac{\gamma t}{4}} \alpha_{-}\left(\omega_{1}\right)\right)\right]\right. \\
& \left.+\left|e^{-\frac{\gamma t}{4}} \alpha_{+}\left(\omega_{1}\right)-e^{\frac{\gamma t}{4}} \alpha_{-}\left(\omega_{1}\right)\right|^{2}\right\} e^{-\frac{t}{4}\left(\widetilde{\gamma}_{11}+\widetilde{\gamma}_{22}^{*}\right)}
\end{aligned}
$$

Hereby, we defined

$$
\begin{aligned}
& \alpha(\widetilde{\omega})=\alpha_{-}(\widetilde{\omega})-\alpha_{+}(\widetilde{\omega}), \\
& \beta(\widetilde{\omega})=\beta_{-}(\widetilde{\omega})-\beta_{+}(\widetilde{\omega}) .
\end{aligned}
$$

Even if both the signal and the idler mode are unexcited initially, i.e.

$$
\left\langle\mathbf{n}_{\text {Signal }}(0)\right\rangle=\left\langle\mathbf{n}_{\text {Idler }}(0)\right\rangle=0,
$$

the signal mode receives quanta by means of spontaneous emission resulting by Eq. (52). Both the down-converted idler noise (Eq. 52) and the noise photons (Eq. 53) represent noise contributions to the signal mode.

In order to obtain the current noise correlation, we need to derive

$c_{11}(\tau)=\left\langle\mathbf{i}_{n 1}(t) \mathbf{i}_{n 1}\left(t^{\prime}\right)\right\rangle$.

The coupling of the LC circuit to the heat bath is a phenomenological method to induce noise and damping into the system, such that the noise currents are not obvious from Eqs. (44a)-(44d). Therefore, we calculate the correlation of

$c_{11}(\tau)=\left\langle\frac{\mathrm{d}}{\mathrm{d} t} \mathbf{Q}_{1}(t) \frac{\mathrm{d}}{\mathrm{d} t} \mathbf{Q}_{1}\left(t^{\prime}\right)\right\rangle$,

and only keep the components representing the induced noise. Following Sargent et al. (1974), we use the expectation values

$$
\begin{aligned}
\left\langle\mathbf{A}_{i \mathrm{H}}(t) \mathbf{g}_{i}^{\dagger}\left(t^{\prime}\right)\right\rangle & =\frac{\tilde{\gamma}_{i i}}{2} \bar{n}\left(\omega_{i}\right), \\
\left\langle\mathbf{A}_{i \mathrm{H}}^{\dagger}(t) \mathbf{g}_{i}\left(t^{\prime}\right)\right\rangle & =\frac{\widetilde{\gamma}_{i i}}{2} \bar{n}\left(\omega_{i}\right), \\
\left\langle\mathbf{g}_{i}^{\dagger}(t) \mathbf{g}_{i}\left(t^{\prime}\right)\right\rangle & =\widetilde{\gamma}_{i i} \bar{n}\left(\omega_{i}\right) \delta\left(t-t^{\prime}\right), \\
\left\langle\mathbf{g}_{i}(t) \mathbf{g}_{i}^{\dagger}\left(t^{\prime}\right)\right\rangle & =\widetilde{\gamma}_{i i}\left[\bar{n}\left(\omega_{i}\right)+1\right] \delta\left(t-t^{\prime}\right), \\
\left\langle\mathbf{g}_{1}(t) \mathbf{A}_{2}^{\dagger}\left(t^{\prime}\right)\right\rangle & =\left\langle\mathbf{g}_{1}^{\dagger}(t) \mathbf{A}_{2}\left(t^{\prime}\right)\right\rangle=0 .
\end{aligned}
$$

The occupation number of the heat bath at initial time is given by the Bose-Einstein statistics

$\bar{n}\left(\omega_{k}\right)=\left\langle\mathbf{b}_{k \mathrm{H}}^{\dagger} \mathbf{b}_{k \mathrm{H}}\right\rangle=\left(e^{\hbar \omega_{k} /\left(k_{\mathrm{B}} T\right)}-1\right)^{-1}$.
The resulting noise current correlation is given by

$c_{11}(\tau)=\frac{\hbar}{\omega_{1} L_{1}} \widetilde{\gamma}_{11}\left(\bar{n}\left(\omega_{1}\right)+\frac{1}{2}\right) \delta\left(t-t^{\prime}\right)$.

One can see, that the coupling of the heat bath to the resonant circuits models noise current resulting from Langevin noise sources. The square-root of the damping constant $\gamma_{1}$ gives the coupling of the Langevin noise source to the circuit, while $\bar{n}\left(\omega_{1}\right)$ is the occupation of the heat bath at resonance frequency $\omega_{1}$. In contrast to the classical Langevin noise source, even if no quanta occupy the heat bath mode with resonance frequency $\omega_{1}$, noise is induced into the circuit. This is an explicit consequence of the quantum mechanical treatment including the vacuum field, approving the need of a quantum mechanical description of the DCPJPA. Comparison of the classical with the quantum mechanical correlation function yields the relation

$$
\frac{4}{G_{1}} \leftrightarrow \frac{V \kappa_{1}^{2}\left(\omega_{1}\right)}{L_{1} \pi^{2} c^{3}} .
$$

The quantum mechanical coupling constant determines the classical, observable conductance.

\section{Numerical Evaluation of the Dynamic Behavior}

We give numerical examples on the dynamics of the expectation value of the signal energy of the DCPJPA described in Eqs. (51)-(53). For comparison with Russer and Russer (2012), we set the frequency of the signal circuit to $f_{1}=$ $2.5 \mathrm{GHz}$ and the idler frequency $f_{2}=\omega_{2} /(2 \pi)=7 \mathrm{GHz}$, and $T$ is chosen as the liquid helium temperature $T=4.2 \mathrm{~K}$.

Figure 2 shows the time evolution of all contribution to the normalized signal mode energy for a high damping. Hereby, we chose the damping constants $\widetilde{\gamma}_{11}=\widetilde{\gamma}_{22}=2 \pi \times 10^{8} \mathrm{~s}^{-1}$ and a Josephson current of $I_{\mathrm{J}}=0.5 \mu \mathrm{A}$. The signal energy decays directly, while the down-converted idler noise rises at the beginning of the consideration. After $3 \mathrm{~ns}$ the idler noise energy decays with the same rate as the signal energy. The noise contribution resulting from the coupling of the heat bath to the signal mode rises all the time, but increases significantly after $40 \mathrm{~ns}$. The lower bound of the total energy is given by $1 / 2 \hbar \omega_{1}$, due to the vacuum fluctuations in the signal mode.

In Fig. 3 the energy terms are represented for a strong driving. Strong driving is achieved, if $\gamma$ exceeds the sum of the damping constants $\widetilde{\gamma}_{11}+\widetilde{\gamma}_{22}$. Here, the pumping by the Josephson junction, indicated by $\gamma_{j}$, is strong enough to overcome the damping. The regime can be controlled by the Josephson current $I_{\mathrm{c}}$. Compared to the settings in Fig. 2, we chose the Josephson current as $I_{\mathrm{c}}=2 \mu \mathrm{A}$. All the energy terms rise exponentially. The signal energy represents the dominating energy contribution, while the down-converted idler noise and the noise induced by the heat bath follow the 


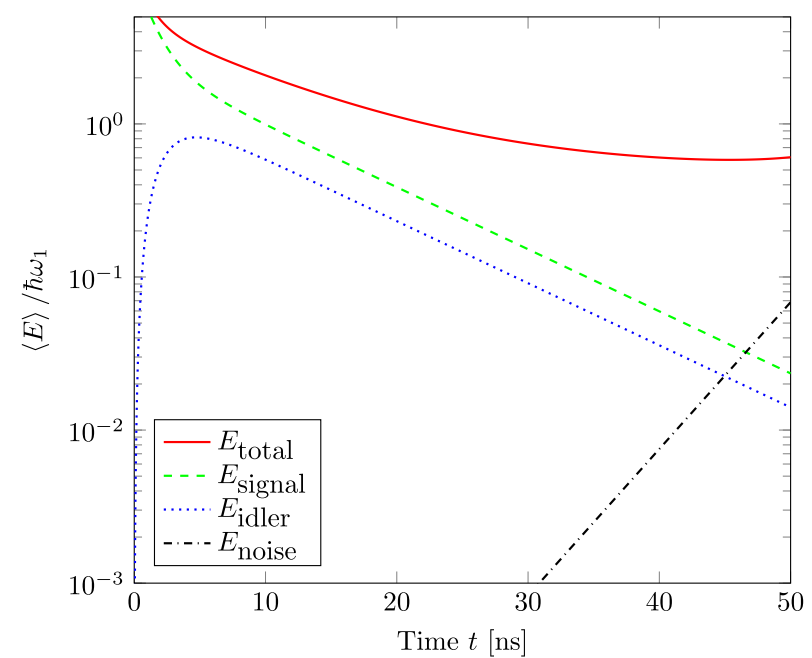

Figure 2. Time evolution of the signal mode energies for strong damping.

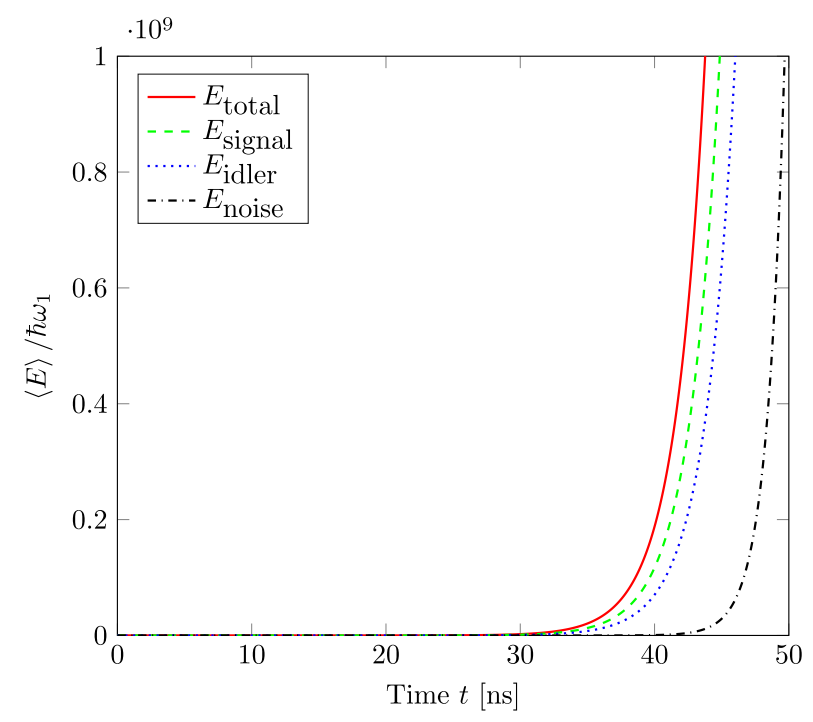

Figure 3. Time evolution of the signal mode energies for strong driving.

signal energy with a delay in time. The energy terms are unbounded, thus the quantum Langevin equations are not capable of modeling any saturation effects in the signal mode. For parametric amplification it is desirable to obtain a steadystate solution. The derived formalism does not yield saturation in the signal mode energy, but rather shows parametric oscillation. Considering Eq. (45), the expectation value of the output field operator increases with rising internal signal mode $\mathbf{A}_{1 \mathrm{H}}$. Driving needs to overcome the dissipation in order to amplify a given signal.

In Fig. 4, we show the temperature dependency of the noise energy as a function of time. A Josephson current of $I_{\mathrm{c}}=1 \mu \mathrm{A}$ is assumed. Figure 4 clearly fulfills the expecta-

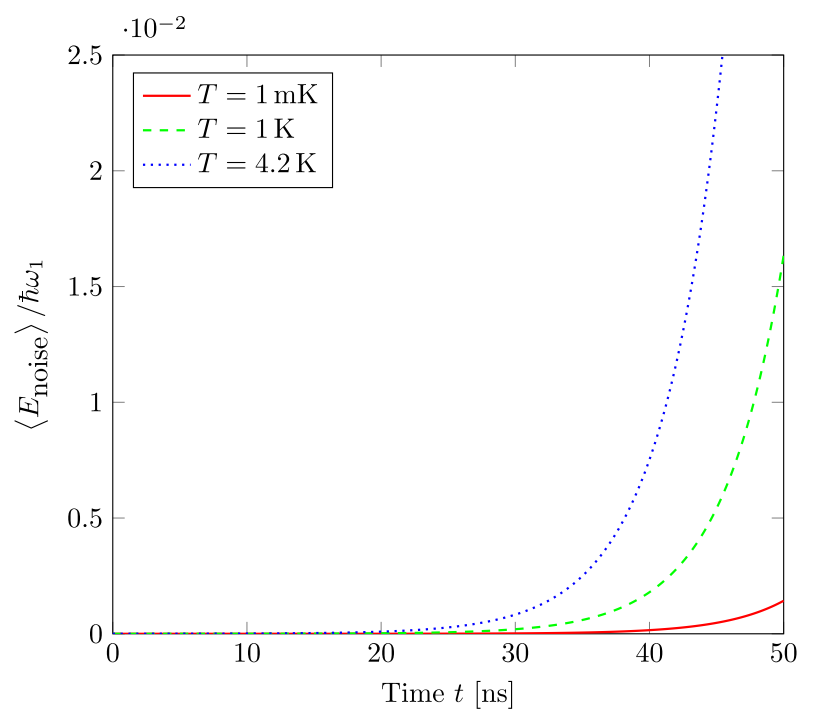

Figure 4. Temperature dependency of the noise energy.

tion, that the noise contribution to the signal mode rises with temperature. Higher temperatures are neglected, as many superconducting materials only contain their superconducting phase below their critical temperature, which for many materials is in the range of liquid helium temperature.

Compared to the dynamics outlined in Russer and Russer (2012), even for the strong driving configuration, the signal energy and the down-converted idler noise are strongly damped. Furthermore, the model derived in Russer and Russer (2012) did not show a decay of the signal energy for strong a damping.

\section{Conclusions}

In this paper, we have investigated the Markovian dynamics of the DC pumped non-degenerate Josephson parametric amplifier. We modeled losses in the DCPJPA using the quantum Langevin approach. Hereby, the resonators, i.e. the signal circuit and the idler circuit, are coupled to a photon heat bath in thermodynamic equilibrium. The DC pump voltage induces an oscillating Josephson current, which is required for coupling of the resonators. We outlined the classical and quantum mechanical model of the lossy DCPJPA and derived the Heisenberg equations of motion. Dissipated signal energy is randomized in the heat bath, for large damping the heat bath injects large noise into the circuit. The expectation value of the signal and noise energies is derived and numerically evaluated for specific settings. Strong damping showed a significant decay of the signal energy and the rise of the noise resulting by the coupling to the heat bath. Moreover, the temperature dependency of the noise energy has been evaluated. The quantum Langevin approach induces dissipation and noise into the dynamical behavior of the DCPJPA, but does not cause saturation in the signal energy. Kaiser et al. 
(2017) give a phenomenological model, in which damping is induced by a multi-photon coupling. Hereby, saturation is reached, but still a theory directly including saturation is desirable. Since many approximations have been used in order to derive an analytic solution, the origin of the missing saturation might be the Markovian assumption itself or the leading order approximation of the cosine in the Josephson Hamiltonian.

Data availability. No data sets were used in this article.

Competing interests. The authors declare that they have no conflict of interest.

Special issue statement. This article is part of the special issue "Kleinheubacher Berichte 2016". It is a result of the Kleinheubacher Tagung 2016, Miltenberg, Germany, 26-28 September 2016.

Acknowledgements. Christian Jirauschek acknowledges funding by the Heisenberg program of the German Research Foundation (DFG, JI115/4-1).

This work was supported by the German Research

Foundation (DFG) and the Technische Universität

München within the funding programme

Open Access Publishing.

Edited by: Dirk Killat

Reviewed by: two anonymous referees

\section{References}

Anderson, P. W. and Rowell, J. M.: Probable Observation of the Josephson Superconducting Tunneling Effect, Phys. Rev. Lett., 10, 230-232, 1963.

Bardeen, J., Cooper, L. N., and Schrieffer, J. R.: Theory of Superconductivity, Phys. Rev., 108, 1175-1204, 1957.

Blackwell, L. A. and Kotzebue, K. L.: Semiconductor-Diode Parametric Amplifiers, Englewood Cliffs, NJ, Prentice Hall, 1961.

de Groot, S. R. and Mazur, P.: Grundlagen der Thermodynamik irreversibler Prozesse, Bibliographisches Institut, Mannheim, Germany, 1969.

Dirac, P. A.: The Quantum Theory of the Emission and Absorption of Radiation, P. Roy. Soc. Lond. A Mat., 114, 243-265, 1927.

Farias, R. L. S., Ramos, R. O., and da Silva, L. A.: Stochastic Langevin Equations: Markovian and NonMarkovian Dynamics, Phys. Rev. E, 80, 031143, https://doi.org/10.1103/PhysRevE.80.031143, 2009.

Feynman, R. P., Leighton, R. B., and Sands, M.: Lectures on Physics: 3 Quantum Mechanics, Addison-Wesley, 1965.

Fock, V.: Konfigurationsraum und zweite Quantelung, Z. Phys., 75, 622-647, 1932.
Gardiner, C. W. and Collett, M. J.: Input and Output in Damped Quantum Systems: Quantum Stochastic Differential Equations and the Master Equation, Phys. Rev. A, 31, 3761-3774, 1985.

Gardiner, C. W. and Zoller, P.: Quantum Noise, Springer, Berlin, 2010.

Haus, H. A.: Electromagnetic Noise and Quantum Optical Measurements, Springer, Berlin, 2000.

Jirauschek, C. and Russer, P.: Hamiltonian Formulations for Lossy Nonlinear Quantum Circuits, in: NDES 2012; Nonlinear Dynamics of Electronic Systems, 1-4, 2012.

Josephson, B. D.: Possible New Effects in Superconductive Tunnelling, Phys. Lett., 1, 251-253, 1962.

Kaiser, W., Haider, M., Russer, J., Russer, P., and Jirauschek, C.: Quantum Theory of the Dissipative Josephson Parametric Amplifier, International Journal of Circuit Theory and Applications, 45, 864-881, 2017.

Kirtley, J. R., Tesche, C. D., Gallagher, W. J., Kleinsasser, A. W., Sandstrom, R. L., Raider, S. I., and Fisher, M. P. A.: Measurement of the Intrinsic Subgap Dissipation in Josephson Junctions, Phys. Rev. Lett., 61, 2372-2375, 1988.

London, F.: Superfluids: Macroscopic Theory of Superconductivity, Dover Publications Inc., vol. 1, 1961.

Louisell, W. H.: Radiation and Noise in Quantum Electronics, McGraw-Hill, New York, 1964.

Macklin, C., O’Brien, K., Hover, D., Schwartz, M., Bolkhovsky, V., Zhang, X., Oliver, W., and Siddiqi, I.: A Near-Quantum-Limited Josephson Traveling-Wave Parametric Amplifier, Science, 350, 307-310, 2015.

Manley, J. M. and Rowe, H. E.: Some General Properties of Nonlinear Elements - Part I, General Energy Relations, Proc. IRE, 44, 904-913, 1956.

Mollow, B. and Glauber, R.: Quantum Theory of Parametric Amplification I, Phys. Rev., 160, 1076-1096, 1967.

Roy, A. and Devoret, M.: Introduction to Parametric Amplification of Quantum Signals with Josephson Circuits, C. R. Phys., 17, 740-755, 2016.

Russer, J. A. and Russer, P.: Lagrangian and Hamiltonian Formulations for Classical and Quantum Circuits, IFAC P. Vol., 45, 439444, 2012.

Russer, P.: Parametric Amplification with Josephson Junctions, Arch. Elektr. Ubertrag., 23, 417-420, 1969.

Russer, P.: General Energy Relations for Josephson Junctions, Proc. IEEE, 59, 282-283, 1971.

Russer, P. and Kaertner, F. X.: Squeezed-State Generation by a DC Pumped Degenerate Josephson Parametric Amplifier, Arch. Elektr. Ubertrag., 44, 216-224, 1990.

Russer, P. and Russer, J. A.: Nanoelectronic RF Josephson Devices, IEEE T. Microw. Theory, 59, 2685-2701, 2011.

Sargent, M., Scully, M. O., and Lamb, W. E.: Laser Physics, Addison-Wesley Press, London, 1974.

Walls, D. F. and Milburn, G. J.: Quantum Optics, Springer Science \& Business Media, 2007.

Wu, Y. and Yang, X.: Strong-Coupling Theory of Periodically Driven Two-Level Systems, Phys. Rev. Lett., 98, 013601, https://doi.org/10.1103/PhysRevLett.98.013601, 2007. 